\title{
Research Paper: Determination of Changes in Blood Biomarker Levels in Antipsychotic Polypharmacy and Aripiprazole Monotherapy in Patients With Long-term Schizophrenia
}

\author{
Venus Vatankhah ${ }^{1}$ (D), Arash Mirabzadeh ${ }^{1,2^{*}}$ (D), Hamidreza Iranpoor ${ }^{3}$ (D), Bahman Dieji ${ }^{1}$ (D), Mehdi Noroozi ${ }^{2}$ (D), Morteza Karimipoor ${ }^{4}$ (D), Jale \\ Nobakht $^{1}$ (i), Elham Esmaeli ${ }^{1}$ (D), Maryam Ayazi ${ }^{1}$ (i) \\ 1. Department of Psychiatry, University of Social Welfare and Rehabilitation Sciences, Tehran, Iran. \\ 2. Social Determinants of Health Research Center, University of Social Welfare and Rehabilitation Sciences, Tehran, Iran. \\ 3. Department of Molecular Biology, Pasteur Institute of Iran, Tehran, Iran \\ 4. Department of Molecular Medicine, Genetics and Metabolism Research Group, Pasteur Institute of Iran, Tehran, Iran
}

\begin{tabular}{|c|c|}
\hline $\begin{array}{l}\text { Use your device to scan } \\
\text { and read the article online }\end{array}$ & Citation: Vatankhah V, Mirabzadeh A, Iranpoor H, Dieji B, Noroozi M, Karimipoor M, et al. Determination of Changes in \\
\hline Q & $\begin{array}{l}\text { Blood Biomarker Levels in Antipsychotic Polypharmacy and Aripiprazole Monotherapy in Patients With Long-term Schizo- } \\
\text { phrenia. Iranian Rehabilitation Journal. 2019; 17(4):369-376. http://dx.doi.org/10.32598/irj.17.4.369 }\end{array}$ \\
\hline 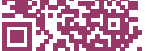 & dol' http://dx.doi.org/10.32598/irj.17.4.369 \\
\hline
\end{tabular}

(c) (i) (8)

Article info:

Received: 07 May 2019

Accepted: 13 Oct 2019

Available Online: 01 Dec 2019

Keywords:

Schizophrenia, Polypharmacy, Aripiprazole, Cytokines

\begin{abstract}
Objectives: Schizophrenia is a chronic psychiatric disorder, which reduces the patient's quality of life. Although a minimum dose of medications has been recommended for treating this disorder, antipsychotic polypharmacy has been used experimentally leading to an increase in drug interactions. Aripiprazole is associated with a lower risk of metabolic side effects and is recommended as a first-line treatment for schizophrenia. Biomarkers can serve as predictive of treatment response in patients with schizophrenia. The aim of this study was to compare the efficacy of antipsychotic medication polypharmacy with Aripiprazole monotherapy in patients with long-term schizophrenia, using blood biomarkers.
\end{abstract}

Methods: Nineteen patients with long-term schizophrenia, who had received at least 2 types of antipsychotics with daily doses of more than $500 \mathrm{mg}$ of Chlorpromazine, were included in the study. The response rates to the treatment based on the Brief Psychiatric Rating Scale (BPRS) score and the blood level of Interleukin 2 (IL-2), Interleukin 6 (IL-6), Interleukin-1 Receptor Antagonist (IL-1RA), and Brain-derived Neurotrophic Factor (BDNF) biomarkers were compared in antipsychotic polypharmacy and 6 months after monotherapy with Aripiprazole.

Results: The mean concentrations of IL-6, IL-1RA, and IL-2 significantly decreased after the intervention. The mean changes in the BPRS scores and also the relationship between changes in blood biomarkers and BPRS scores after intervention were not significant.

Discussion: The conversion of the antipsychotic polypharmacy state to monotherapy with Aripiprazole has been accompanied by a significant decrease in the serum levels of IL-2, IL- 6 , and IL-1RA. These biomarkers can be used for evaluating the response rate of schizophrenia treatments in the future. 


\section{Highlights}

- Interleukin-1 receptor antagonist is an appropriate biomarker for evaluating the response rates in patients with schizophrenia.

- Interleukin 2 is an appropriate biomarker for evaluating the response rates in patients with schizophrenia.

- Interleukin 6 is an appropriate biomarker for evaluating the response rates in patients with schizophrenia.

\section{Plain Language Summary}

In the future, psychiatrists can use the appropriate pattern of treatment for patients to measure the serum level of blood biomarkers to follow the treatment of patients with schizophrenia. The Interleukin-1 Receptor Antagonist (IL-1RA), interleukin-2, and interleukin-6 are probably biomarkers for considering response rates in patients with schizophrenia.

\section{Introduction}

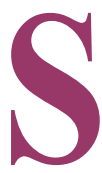

chizophrenia is a psychiatric disorder with a wide range of biological and clinical manifestations. It affects $1 \%$ of the population; however, its exact causative factor is not completely known [1]. More than $50 \%$ of people diagnosed with schizophrenia have intermittent and long-term psychiatric problems and about $20 \%$ of chronic symptoms and disabilities are developed [2]. Generally, in all available schizophrenia guidelines, drug monotherapy is recommended, and as the last resort, using two or more antipsychotics is suggested [3, 4]. The disadvantages of antipsychotic polypharmacy are as follow: 1. The use of two or more medications with inducing and inhibiting effects in the cytochrome P450 system that can lead to drug interactions [5]; 2. An excessive increase in the dosage of antipsychotics in the patient and subsequently leading to dose-related adverse effects [6]; 3. An increase in the cost of patient treatment [7]; 4. The increased likelihood of death in patients [8]; 5 . The discouragement of the patient from taking regular medications [9].

Given the unique mechanism of Aripiprazole, this medicine partially or completely reduces the metabolic side effects of antipsychotics and can decrease the prolactin [10]. Aripiprazole has a unique pharmacological effect, which is associated with a lower risk of hyperprolactinemia, metabolic complications, weight gain, increased cholesterol, and high blood pressure; it is also widely used as the first-line therapy for schizophrenia $[11,12]$. Biomarkers can serve as the predictors of diagnosis and response to treatment in patients with schizophrenia and result in advancements in new therapeutic methods for schizophrenia. Since brain biopsy is not fea- sible in the patient and also brain imaging techniques are costly, they are not applicable in identifying and tracking schizophrenia. The identification of biomarkers, which can be used as a diagnostic or predictive factor for response to treatment in people with schizophrenia, is an important step in the ability to provide personalized treatment and attempt to develop new therapies. In addition, the high degree of heritability in schizophrenia suggests that genetic markers may be detectable in the peripheral tissue. The Brain-derived Neurotrophic Factor (BDNF) is the most commonly distributed neuronal agent in the brain, which level is associated with schizophrenia. Hypermethylation of the BDNF gene promoter in patients with schizophrenia is reported [13].

The cytokines, such as Interleukin 2 (IL-2) and Interleukin 6 (IL-6) are altered in the serum of schizophrenic patients with an upstream regulation of inflammatory cytokines and some of their receptors, such as anti-inflammatory Interleukin-1 Receptor Antagonist (IL-1RA) [14]. By determining the rate of using antipsychotic drugs in patients with chronic schizophrenia in the Razi Psychiatric Hospital and the relationship between the type of treatment and the studied variables, as well as the results of the research, all physicians can be expected to use the appropriate pattern of treatment in the future for patients to measure the serum level of blood biomarkers to follow the treatment of patients with schizophrenia. In this study, by measuring the specific blood biomarkers to provide follow-up therapy for schizophrenia, the response to treatment was compared in the antipsychotic medication polypharmacy and Aripiprazole monotherapy groups. 


\section{Methods}

This clinical trial (without a control group) was carried out before and after intervention on the case group (patients with schizophrenia). Of all patients with long-term schizophrenia (more than 2 years), who were referred to the long-term admission departments of the Razi Psychiatric Hospital, all who received at least 2 types of antipsychotics with daily doses of more than $500 \mathrm{mg}$ of Chlorpromazine for more than 6 months, enrolled in the study. The inclusion criteria included patients aged 18 to 65 years with long-term schizophrenia (more than 2 years), who received at least 2 antipsychotic drugs with a dose of more than $500 \mathrm{mg}$ of chloramphenicol per day.

The exclusion criteria also included autoimmune diseases, uncontrolled diabetes, cardiovascular diseases, severe infectious disease requiring serious intervention, treatment with immunosuppressive drugs, the use of antibiotics within 1 month before evaluating the serum biomarker level, other severe brain disorders, such as seizures and multiple sclerosis, mental disability, alcohol or drug abuse, the simultaneous presence of other major psychiatric illnesses requiring drug therapy, the exacerbation of the symptoms that conflicts with the clinical trials, and unwillingness to continue the study. At the beginning of the study, the following factors were determined: the patients' age and sex, the type and amount of all used psychiatric drugs (especially antipsychotics, as well as the doses of Chlorpromazine equivalent), the total duration of the disease, and the duration of the polypharmacy treatment. After obtaining consent from the patients or their legal guardians, they were placed in the clinical trial stage. Before the intervention, the Brief Psychiatric Rating Scale (BPRS) score, as well as the serum level of IL-2, IL-6, IL-1RA, and BDNF, were measured. The intervention included the conversion of antipsychotic medication polypharmacy to monotherapy with Aripiprazole.

Each $100 \mathrm{mg}$ of the Chlorpromazine was considered as $7.7 \mathrm{mg}$ of Aripiprazole. With regard to the recurrence rate, the number of previous drugs gradually decreased and the administration of Aripiprazole initiated at a dose of $2.5 \mathrm{mg}$. Finally, a total dose was calculated and the maximum dose prescribed for the drug was administrated. Based on the clinical status of the patient the occurrence of drug complications, the process of changes in drug dose was lowered or stopped. After reaching the Aripiprazole monotherapy phase, the treatment lasted for 6 months, during which the patients were visited regularly by a psychiatric assistant to check clinical signs and side effects. After 6 months of treatment with Ar- ipiprazole monotherapy, the BPRS score, as well as the serum levels of IL-2, IL-6, IL-1RA, and BDNF, were compared to the baseline. The sample size was calculated 13 subjects based on the study of Lawrence S Kegeles et al. with 95\% confidence interval, 90\% statistical power, mean difference of 4.1, Standard Deviation (SD) of 4.3 , a probable loss of $15 \%$, and the mean comparison formula. IL-2, IL-6, IL-1RA, and BDNF were measured by ELISA kits (Abcam Co. UK) [15].

The mean and SD were reported for quantitative analysis and the frequency tables were reported for frequency variables. For data analysis, the correlation analysis and paired t-test were used. Statistical analyses were performed via SPSS V. 16.

\section{Results}

In this study, 26 patients with schizophrenia, who had the inclusion criteria, were included; 7 of them (4 women and 3 men) were excluded during the intervention and, finally, 19 patients were studied. 15 subjects (78.9\%) were male and 4 subjects $(21.1 \%)$ were female with the Mean \pm SD age of $49 \pm 49.49$ years (age range of 33-65 years). The Mean \pm SD age of male patients was $50.46 \pm 7.43$ years (age range of 38-60 years), whereas the Mean \pm SD age of female patients was $45.33 \pm 12.72$ years (age range of 33-65 years). The Mean \pm SD duration of schizophrenia was $17.55 \pm 11.47$ years (age range of 2-34 years). The Mean \pm SD duration of polypharmacy therapy was $3.42 \pm 2.93$ years ranging from $0.5-16$ years. Moreover, the Mean \pm SD total dose of polypharmacy antipsychotics based on the dose of Chlorpromazine before the onset of the intervention was $963.68 \pm 43.69$ ranging from $500 \mathrm{mg}$ to $1860 \mathrm{mg}$. The paired t-test was used to measure blood biomarker concentrations (Table 1).

Plasma concentrations of IL-6, IL-1RA, and IL-6 significantly decreased from polypharmacy to monotherapy with Aripiprazole after changing the antipsychotic drug $(\mathrm{P}<0.05)$. The concentration of BDNF biomarker increased after the intervention; however, this increase was not significant $(\mathrm{P}=0.734)$. There was no significant difference in the level of blood biomarkers between men and women; in both groups, the plasma concentrations of IL-6, IL-1RA, and IL-2 were significant after the intervention. The patients were divided into 2 groups of less than 50 years old and over 50 years old based on homogeneous distribution Table 2 and blood biomarkers were compared in these groups. In patients older than 50 years, IL-6, IL-1RA, and IL-2 were significantly decreased after the intervention $(\mathrm{P}<0.05)$. 
Table 1. Serum level of biomarkers in two regimens of antipsychotic polypharmacy and monotherapy with Aripiprazole in schizophrenic patients

\begin{tabular}{|c|c|c|c|c|}
\hline \multirow{2}{*}{ Variables } & \multicolumn{2}{|c|}{ Mean $\pm S D$} & \multirow{2}{*}{$\mathbf{T}$} & \multirow{2}{*}{$\mathbf{P}$} \\
\hline & Before Intervention & After Intervention & & \\
\hline IL-2 & $2.898 \pm 0.908$ & $2.100 \pm 0.584$ & 3.023 & 0.007 \\
\hline IL-1RA & $181.401 \pm 5.219$ & $174.026 \pm 5.127$ & 4.194 & $0.001>$ \\
\hline IL-6 & $8.61 \pm 1.11$ & $7.02 \pm 0.97$ & 8.525 & $0.001>$ \\
\hline BDNF & $5.001 \pm 1.007$ & $5.114 \pm 1.964$ & -0.345 & 0.734 \\
\hline
\end{tabular}

Iranian Rehabilitation \ournal

Table 2. Significant changes in blood biomarkers before and after the intervention based on age

\begin{tabular}{ccccc}
\hline $\begin{array}{c}\text { Biomarker } \\
\text { Age }(\mathbf{y})\end{array}$ & IL-2 & IL-1RA & IL-6 & BDNF \\
\hline Less than 50 & 0.138 & 0.014 & $0.001>$ & 0.734 \\
\hline More than 50 & 0.011 & 0.004 & $0.001>$ & 0.967 \\
\hline
\end{tabular}

Iranian Rehabilitation』ournal

Table 3. Significant changes in blood biomarkers before and after the intervention based on the equivalent dose of Chlorpromazine

\begin{tabular}{ccccc}
\hline $\begin{array}{c}\text { Biomarker } \\
\text { Dose Chlorpromazine (mg) }\end{array}$ & IL-2 & IL-1RA & IL-6 & BDNF \\
\hline Less than 700 & 0.938 & 0.005 & 0.009 & 0.391 \\
$700-1000$ & 0.134 & 0.19 & 0.008 & 0.484 \\
\hline More than 1000 & 0.006 & 0.002 & $0.001>$ & 0.163 \\
\hline
\end{tabular}

Iranian Rehabilitation \ourna

The subjects were assigned to 3 groups based on the equivalent dose of Chlorpromazine that they received; their changes in blood biomarkers were also monitored (Table 3). IL-6 and IL-1RA concentrations were significantly reduced in patients with previous treatment with a dose of less than $700 \mathrm{mg}$ of Chlorpromazine after the intervention $(\mathrm{P}<0.05)$. The changes in IL-6 concentrations in patients with previous therapeutic regimens with a dose of $700 \mathrm{mg}$ to $1000 \mathrm{mg}$ Chlorpromazine had a significant decrease $(\mathrm{P}=0.008)$. Changes in the concentration of IL-6, IL-1RA, and IL-2 were significantly reduced in patients, who had polypharmacy with a dose of more than $1000 \mathrm{mg}$ of Chlorpromazine $(\mathrm{P}<0.05)$.

Patients were divided into 4 groups based on the duration of the disorder, and changes in their blood bio-

Table 4. Significant changes in blood biomarkers before and after the intervention based on the duration of the disorder

\begin{tabular}{cccccc}
\hline $\begin{array}{c}\text { Interleukin } \\
\text { Duration of the Disorder }(\mathbf{y})\end{array}$ & IL-2 & IL-1RA & IL-6 & BDNF \\
\hline Less than 10 & 0.800 & 0.164 & 0.003 & 0.092 \\
Between 10 and 20 & 0.018 & 0.059 & 0.002 & 0.126 \\
Between 20 and 30 & 0.101 & 0.056 & 0.046 & 0.537 \\
More than 30 & 0.099 & 0.043 & 0.021 & 0.860 \\
\hline
\end{tabular}


Table 5. Significant changes in blood biomarker levels after intervention based on the times in the polypharmacy status

\begin{tabular}{ccccc}
\hline $\begin{array}{c}\text { Interleukin } \\
\text { Duration of Polypharmacy }(\mathbf{y})\end{array}$ & IL-2 & IL-1RA & IL6 & BDNF \\
\hline Less than 2 & 0.01 & 0.001 & 0.002 & 0.539 \\
More than 2 & 0.05 & 0.021 & $0.001>$ & 0.969 \\
\hline
\end{tabular}

Iranian Rehabilitation Journa

Table 6. The correlation between the changes in serum levels of biomarkers after intervention and the BPRS scores of patients

\begin{tabular}{ccc}
\hline Variable & Correlation & P \\
\hline Changes in Interleukin 2 and BPRS & 0.21 & 0.37 \\
\hline Changes in Interleukin 1RA and BPRS & 0.2 & 0.3 \\
Changes in Interleukin 6 and BPRS & 0.011 & 0.96 \\
BDNF and Changes in BPRS & -0.20 & 0.4 \\
\hline
\end{tabular}

Iranian Rehabilitation \ourna

markers were compared (Table 4). In patients, who had schizophrenia for less than 10 years, the concentration of IL-6 significantly decreased ( $\mathrm{P}=0.003)$. IL-6, IL-1RA, and IL-2 concentrations significantly decreased in those, who had experienced schizophrenia for 10 to 20 years $(\mathrm{P}<0.05)$. In patients, who had schizophrenia for over 30 years, the IL- 6 and IL-1RA concentrations were significantly reduced after the intervention $(\mathrm{P}<0.05)$. However, changes in BDNF and IL-2 were not significant in this group of patients. The subjects were divided into 2 groups based on the times in the polypharmacy status, and the changes in their blood biomarkers were compared (Table 5). In patients with polypharmacy of fewer than 2 years, a significant decrease was observed in the concentrations of IL-2, IL-6, and IL-1RA $(\mathrm{P}<0.05)$. In patients with polypharmacy longer than 2 years, the IL2, IL-6, and IL-1RA concentrations were significantly reduced $(\mathrm{P}<0.05)$.

The Mean \pm SD BPRS score of the subjects at the baseline and after the intervention was $25.736 \pm 10.521$ and $12 \pm 26.6$, respectively. There was no significant difference in the BPRS score in cases before and after the intervention (Table 6). The results of the Pearson correlation test indicated a positive correlation between IL-6, IL-1RA, IL-2, and BPRS changes; however, this was not statistically significant $(\mathrm{P}>0.05)$. In addition, there was no significant difference in BDNF concentration and BPRS scores after the intervention $(\mathrm{P}>0.05)$.

\section{Discussion}

Generally, $78.9 \%$ of patients with schizophrenia were male. The prevalence of schizophrenia is equivalent for men and women; however, the onset and severity of disorder are different in both sexes. On the other hand, in previous studies, there has been more antipsychotic polypharmacy in males than females; it can be as a result of the physician's perceptions that basically male patients are expected to be more severely agitated and aggressive than females. In addition, possibly men are physically more able to tolerate combination therapy [16].

In this study, 8 women were studied, which was significantly less than the number of male patients. Therefore, variations in variables cannot be considered for gender differences, which is one of the limitations of this study. In the present study, the level of IL-6, IL1RA, and IL-2 after the intervention was significantly reduced. Based on the results of previous studies on changes in the level of interleukins, schizophrenia has been shown to increase plasma levels of these interleukins and they are reduced after treatment. Regarding the lower level of these interleukins in the treatment of Aripiprazole monotherapy compared to polypharmacy, it can be concluded that monotherapy with Aripiprazole provides better control of the disease. In 2010, RC Drexhage et al. showed that the levels of IL-2 and IL-6 in schizophrenic patients were, respectively, increased and decreased in treatment duration, which is consistent with the results of this study [17]. 
The results of a study by Leffler in 2010 showed that in the long term, the plasma IL-6 levels decreased in patients treated with Clozapine monotherapy. The results of this study, as well as our results, indicate that the new generation antipsychotics are associated with a reduction in IL-6 levels. Comparing the results of this study and the results of the current research, treatment with Aripiprazole has been associated with a higher reduction in IL-6 levels than Clozapine treatment; the reason might be because of the use of Clozapine in patients resistant to treatment, who were less affected by the level of IL-6 associated with treatment. In this study, changes in the level of blood biomarkers were compared with the age groups of less than 50 years and over 50 years. In patients younger than 50 years old, only the plasma levels of IL-6 and IL-1RA significantly decreased. In patients older than 50 years, changes in IL-6, IL-1RA, and IL-2 were significantly decreased; it indicated that IL-6 and IL-1RA are linked to the more desirable results than IL-2 to assess the response to treatment in patients with schizophrenia at an early age [18].

In the present study, serum levels of BDNF significantly increased after intervention than baseline levels, but this increase was not significant. Lai et al. reported a significant increase in serum BDNF levels following better control of schizophrenia. According to Fernandes, serum BDNF levels have no positive relationship with schizophrenia [19]. Green et al. research entitled "BDNF levels in schizophrenia: A systematic review of metaanalysis" showed that the Neurotrophin levels decreased in patients with schizophrenia. Regarding the contradictory results of the plasma level of BDNF in patients with schizophrenia and the fact that this biomarker is changed in many diseases other than schizophrenia, including mood disorders, it can be concluded that BDNF has less specificity for schizophrenia than other biomarkers. BDNF measurement is less relevant than other biomarkers in order to evaluate the response to treatment in patients with schizophrenia [20]. In a study conducted in 2015, in which Aripiprazole increased the plasma levels of brain neurotrophic factors, there was a significant negative correlation between the duration of the disorder and plasma BDNF level [21].

In the present study, the plasma concentration of BDNF increased in the groups based on the duration of the disorder, but these changes were not significant. Patients with polypharmacy in all 3 groups received an antipsychotic medication dose based on the dose of Chlorpromazine and they found a significant decrease in plasma IL-6 levels. This finding suggests that changes in IL-6 are more relevant than other interleukins to assess the response rate in patients with schizophrenia. Changes in IL-2 levels were only significant in the patients treated with polypharmacy with a dose of more than $1000 \mathrm{mg}$ of Chlorpromazine; it indicated the efficacy of this biomarker in assessing the response to treatment in patients with severe schizophrenia, who uses the higher doses of antipsychotics. Studying the changes in blood biomarkers based on the duration of the disorder in patients, who had schizophrenia for less than 10 years, only changes in the IL- 6 biomarker were significant.

This finding can indicate that IL-6 biomarker is more suitable for assessing response to treatment in patients with schizophrenia. In the patients with disorder duration of 10 to 20 years, the changes in IL-2, IL-6, and IL-1RA were observed. In the schizophrenia group with disease duration of 20 to 30 years, only a reduction in the IL-1 biomarker was found. Moreover, in the schizophrenic group with disorder more than 30 years, the levels of IL-6 and IL-1RA significantly decreased. These findings suggest that IL-1RA can be an appropriate biomarker for examining the response to treatment in patients with schizophrenia. Blood biomarker level changes in 2 groups of patients with a duration of more than 2 years and less than 2 years in a polymorphic condition were not significantly different. Therefore, in this study, the duration of the polypharmacy was not effective on the level of blood biomarker levels.

We also evaluated the response rate to treatment based on the reduction of clinical symptoms in the BPRS questionnaire. The Mean \pm SD BPRS baseline score in patients was $25.736 \pm 10.531$, which changed to $26 \pm 12.6$ after treatment and it was not statistically significant. Accordingly, there was no clinically significant reduction in the symptoms of patients with schizophrenia, which can be because of the short study duration to examine the changes in symptoms. In 2014, Mirabzadeh et al. in a clinical trial changed the pattern of taking antipsychotics from polypharmacy to monotherapy with Risperidone in elderly patients with long-term schizophrenia [22]. The results of this study showed a significant reduction in the mean scores of the total and positive schizophrenic symptoms based on the BPRS test after treatment with Risperidone.

The difference in the results of the present study and the mentioned study in reducing the symptoms of patients after changing to the Aripiprazole monotherapy can be attributed to the age differences of patients in two studies. It seems that elderly patients are more susceptible to drug side effects of antipsychotic polypharmacy, and after changing to antipsychotic monotherapy, their BPRS 
score significantly decreased. In this study, the relationship between BPRS score changes, as well as changes in the level of each blood biomarker, was examined before and after the intervention. There was a positive relationship between changes in BPRS score and IL-6, IL-6, and IL-1RA. By decreasing the disorder symptoms in the BPRS questionnaire, the level of these biomarkers was also decreased; however, this relationship was not statistically significant, which is consistent with the results of previous studies.

In this study, there was a negative correlation between BDNF plasma level changes and patient scores in the BPRS test. By increasing the concentration of BDNF, the overall score of the BPRS test was reduced; however, these changes were not statistically significant [22]. A meta-analysis in 2013 showed that plasma BDNF levels were not related to the number of positive or negative symptoms in people with schizophrenia, which is consistent with the findings of this study. This meta-analysis also showed that plasma BDNF had a positive relationship with cognitive function (for example, semantic production tasks) and hearing processing after computer cognitive training in patients [23]. In the present research, a short follow-up was considered for patients in antipsychotic monotherapy, which could affect the BPRS results. A similar study with a longer follow-up is suggested. In this study, the number of women, who had the inclusion criteria, was less than the number of men, which reduced the validity of the results in terms of gender. It is recommended that studies with larger sample size and proper sexual distribution be carried out in the future.

\section{Conclusion}

By determining the pattern of antipsychotic drug use in patients with chronic schizophrenia in the women and men's wards in Razi Psychiatric Hospital and also because of the relationship between the type of treatment, studied variables, and the obtained results, it can be expected that in the future, using the appropriate therapeutic method, the serum level of blood biomarkers can be measured to follow-up the treatment of patients with schizophrenia disorder. The IL-1RA, IL-2, and IL-6 are probably biomarkers for assessing the response to treatment in patients with schizophrenia, which requires further studies with a larger sample size for confirmation. In this study, the changes in the plasma level of these biomarkers did not correlate with the response rate of patients to treatment based on the BPRS questionnaire.

\section{Ethical Considerations}

\section{Compliance with ethical guidelines}

The study was approved by the Ethics Committee of the University of Social Welfare and Rehabilitation Sciences (IR.USWR.REC.1396.154).

\section{Funding}

The present research was extracted from the thesis written by Venus Vatankhah and was financially supported by the National Institute for Medical Research Development (Grants No: 973020).

\section{Authors contributions}

Methodology: Venus Vatankhah, Hamidreza Iranpour, Morteza Karimipoor, Mehdi Noroozi, Bahman Dieji, Jale Nobakht, Elham Esmaeli, Maryam Ayazi; Investigation: Ararsh Mirabzadeh; Writing-original draft: Venus Vatankhah, Hamidreza Iranpour. Writing-review \& editing: Ararsh Mirabzadeh, Mehdi Noroozi; Funding acquisition: Ararsh Mirabzadeh; Supervision: Arash Mirabzadeh.

\section{Conflict of interest}

The authors declared no conflict of interest.

\section{Refrences}

[1] Emsley R, Chiliza B, Asmal L, Harvey BHJBp. The nature of relapse in schizophrenia. BMC Psychiatry. 2013; 13(1):50. [DOI:10.1186/1471-244X-13-50] [PMID] [PMCID]

[2] Barbato AJA, Psychiatry NZJo. Psychiatry in transition: Outcomes of mental health policy shift in Italy. Australian and New Zealand Journal of Psychiatry. 1998; 32(5):673-9. [DOI:10.3109/00048679809113122] [PMID]

[3] Canadian Psychiatric Association. Clinical practice guidelines. Treatment of schizophrenia. The Canadian Journal of Psychiatry. 2005; 50(13 Suppl):7S-57S. [PMID]

[4] Crismon ML, Argo TR, Bendele BS, Suppes T. Texas medication algorithm project procedural manual. Bipolar Disorder Algorithms. The Texas Department of State Health Services. 2007.

[5] Jeste DV, Caligiuri MP, Paulsen JS, Heaton RK, Lacro JP, Harris MJ, et al. Risk of tardive dyskinesia in older patients: A prospective longitudinal study of 266 outpatients. Archives of General Psychiatry. 1995; 52(9):756-65. [DOI:10.1001/ archpsyc.1995.03950210050010] [PMID]

[6] Sakurai H, Bies RR, Stroup ST, Keefe RS, Rajji TK, Suzuki $\mathrm{T}$, et al. Dopamine D2 receptor occupancy and cognition in 
schizophrenia: Analysis of the CATIE data. Schizophrenia Bulletin. 2012; 39(3):564-74. [DOI:10.1093/schbul/sbr189] [PMID] [PMCID]

[7] Urichuk L, Prior TI, Dursun S, Baker GJCdm. Metabolism of atypical antipsychotics: Involvement of cytochrome p450 enzymes and relevance for drug-drug interactions. Current Drug Metabolism. 2008; 9(5):410-8. [DOI:10.2174/1389200087 84746373] [PMID]

[8] Ray WA, Chung CP, Murray KT, Hall K, Stein CM. A typical antipsychotic drugs and the risk of sudden cardiac death The New England Journal of Medicine .2009; 360(3):225-35. [DOI:10.1056/NEJMoa0806994] [PMID] [PMCID]

[9] Benner JS, Chapman RH, Petrilla AA, Tang SS, Rosenberg N, Schwartz JSJAJoH-SP. Association between prescription burden and medication adherence in patients initiating antihypertensive and lipid-lowering therapy. American Journal of Health-System Pharmacy. 2009; 66(16):1471-7. [DOI:10.2146/ ajhp080238] [PMID]

[10] Chen CK, Huang YS, Ree SC, Hsiao CCJPiNp, Psychiatry B. Differential add-on effects of aripiprazole in resolving hyperprolactinemia induced by risperidone in comparison to benzamide antipsychotics. Neuro-Psychopharmacology and Biological Psychiatry. 2010; 34(8):1495-9. [DOI:10.1016/j.pnpbp.2010.08.012] [PMID]

[11] Leucht S, Cipriani A, Spineli L, Mavridis D, Örey D, Richter F, et al. Comparative efficacy and tolerability of 15 antipsychotic drugs in schizophrenia: A multiple-treatments meta-analysis. Focus. 2014; 12(2):192-204. [DOI:10.1176/appi. focus.12.2.192]

[12] Rummel-Kluge C, Komossa K, Schwarz S, Hunger H, Schmid F, Kissling W, et al. Second-generation antipsychotic drugs and extrapyramidal side effects: A systematic review and meta-analysis of head-to-head comparisons. Schizophrenia Bulletin. 2010; 38(1):167-77. [DOI:10.1093/schbul/sbq042] [PMID] [PMCID]

[13] Fernandes B, Berk M, Turck C, Steiner J, Goncalves CJMp. Decreased peripheral brain-derived neurotrophic factor levels are a biomarker of disease activity in major psychiatric disorders: A comparative meta-analysis. Molecular Psychiatry. 2014; 19(7):750-1. [DOI:10.1038/mp.2013.172] [PMID]

[14] de Witte L, Tomasik J, Schwarz E, Guest PC, Rahmoune $\mathrm{H}$, Kahn RS, et al. Cytokine alterations in first-episode schizophrenia patients before and after antipsychotic treatment. Schizophrenia Research. 2014; 154(1-3):23-9. [DOI:10.1016/j. schres.2014.02.005] [PMID]

[15] Kegeles LS, Slifstein M, Xu X, Urban N, Thompson JL, Moadel T, et al. Striatal and extrastriatal dopamine D2/D3 receptors in schizophrenia evaluated with $[18 \mathrm{~F}]$ fallypride positron emission tomography. Biological Psychiatry. 2010 68(7):634-41. [DOI:10.1016/j.biopsych.2010.05.027] [PMID] [PMCID]

[16] Stahl SM, Grady MM. High-cost use of second-generation antipsychotics under California's Medicaid program Psychiatric Services. 2006; 57(1):127-9. [DOI:10.1176/appi. ps.57.1.127] [PMID]

[17] Drexhage RC, Knijff EM, Padmos RC, Heul-Nieuwenhuijzen Lvd, Beumer W, Versnel MA, et al. The mononuclear phagocyte system and its cytokine inflammatory networks in schizophrenia and bipolar disorder. Journal Expert Re- view of Neurotherapeutics. 2010; 10(1):59-76. [DOI:10.1586/ ern.09.144] [PMID]

[18] Löffler S, Klimke A, Kronenwett R, Kobbe G, Haas R, Fehsel KJJocp. Clozapine mobilizes CD34+ hematopoietic stem and progenitor cells and increases plasma concentration of interleukin 6 in patients with schizophrenia. Psychiatry Servises. 2010; 30(5):591-5. [DOI:10.1097/JCP.0b013e3181eeb7f7] [PMID]

[19] Fernandes BS, Molendijk ML, Köhler CA, Soares JC, Leite CMG, Machado-Vieira R, et al. Peripheral Brain-Derived Neurotrophic Factor (BDNF) as a biomarker in bipolar disorder: A meta-analysis of 52 studies. BMC Medicine. 2015; 13(1):1-22. [DOI:10.1186/s12916-015-0529-7] [PMID] [PMCID]

[20] Green M, Matheson S, Shepherd A, Weickert C, Carr VJMp. Brain-derived neurotrophic factor levels in schizophrenia: A systematic review with meta-analysis. Molecular Psychiatry. 2011; 16(9):960-72. [DOI:10.1038/mp.2010.88] [PMID]

[21] Yoshimura R, Hori H, Ikenouchi-Sugita A, Umene-Nakano W, Katsuki A, Hayashi K, et al. Aripiprazole altered plasma levels of brain-derived neurotrophic factor and catecholamine metabolites in first-episode untreated Japanese schizophrenia patients. Human Psychopharmacology. 2012; 27(1):33-8. [DOI:10.1002/hup.1257] [PMID]

[22] Mirabzadeh A, Shemshadi H, Khodaei MR, Sadighi G, Hashempour S, Mirabzadeh H, et al. Antipsychotic polypharmacy versus monotherapy in elderly patients with chronic schizophrenia: A clinical trial. Journal Klinik Psikofarmakoloji Bülteni-Bulletin of Clinical Psychopharmacology. 2014; 24(4):302-10. [DOI:10.5455/bcp.20140701043605]

[23] Ikegame T, Bundo M, Sunaga F, Asai T, Nishimura F, Yoshikawa A, et al. DNA methylation analysis of BDNF gene promoters in peripheral blood cells of schizophrenia patients Neuroscience Research. 2013; 77(4):208-14. [DOI:10.1016/j. neures.2013.08.004] [PMID] 